\title{
DIFFERENCE METHODS FOR BOUNDARY VALUE PROBLEMS IN ORDINARY DIFFERENTIAL EQUATIONS*
}

\author{
H. B. KELLER $\dagger$ AND A. B. WHITE, JR.
}

Abstract. A general theory of difference methods for problems of the form

$$
\mathscr{N} \mathbf{y} \equiv \mathbf{y}^{\prime}-\mathbf{f}(t, \mathbf{y})=\mathbf{0}, \quad a \leqq t \leqq b, \quad \mathbf{g}(\mathbf{y}(a), \mathbf{y}(b))=\mathbf{0},
$$

is developed. On nonuniform nets, $t_{0}=a, t_{j}=t_{j-1}+h_{j}, 1 \leqq j \leqq J, t_{J}=b$, schemes of the form

$$
\mathscr{N}_{h} \mathbf{u}_{j} \equiv \mathbf{G}_{j}\left(\mathbf{u}_{0}, \cdots, \mathbf{u}_{J}\right)=\mathbf{0}, \quad 1 \leqq j \leqq J, \quad \mathbf{g}\left(\mathbf{u}_{0}, \mathbf{u}_{J}\right)=\mathbf{0}
$$

are considered. For linear problems with unique solutions, it is shown that the difference scheme is stable and consistent for the boundary value problem if and only if, upon replacing the boundary conditions by an initial condition, the resulting scheme is stable and consistent for the initial value problem. For isolated solutions of the nonlinear problem, it is shown that the difference scheme has a unique solution converging to the exact solution if (i) the linearized difference equations are stable and consistent for the linearized initial value problem, (ii) the linearized difference operator is Lipschitz continuous, (iii) the nonlinear difference equations are consistent with the nonlinear differential equation. Newton's method is shown to be valid, with quadratic convergence, for computing the numerical solution.

1. Introduction. We present a new and rather comprehensive theory of general difference methods for approximating the solution of both linear and nonlinear boundary value problems for first order systems of ordinary differential equations. For linear problems with unique solutions our theory, in $\S 3$, states essentially that a difference scheme is stable and consistent for the boundary value problem if and only if it is stable and consistent for the initial value problem. For isolated solutions of nonlinear problems our theory, in $\S 4$, states that a difference scheme has a unique solution converging to the isolated solution if (i) the linearized difference equations are stable and consistent for the linearized initial value problem, (ii) the linearized difference operator is Lipschitz continuous, (iii) the nonlinear difference equations are consistent with the nonlinear problem. Newton's method is shown to be valid, with quadratic convergence, for computing the numerical solution.

The linear boundary value problems that we study include the general form

$$
\begin{gathered}
\mathscr{L} \mathbf{y} \equiv \mathbf{y}^{\prime}-A(t) \mathbf{y}=\mathbf{f}(t), \quad a \leqq t \leqq b, \\
\mathscr{B} \mathbf{y} \equiv B_{a} \mathbf{y}(a)+B_{b} \mathbf{y}(b)=\boldsymbol{\beta} .
\end{gathered}
$$

Here $\mathbf{y}(t), \mathbf{f}(t)$ and $\boldsymbol{\beta}$ are $n$-vectors, $A(t), B_{a}$ and $B_{b}$ are $n \times n$ matrices; the elements of $A(t)$ and $\mathbf{f}(t)$ are in $C^{N}[a, b]$ while the solution $\mathbf{y}(t)$ is in $C^{N+1}[a, b]$. With little extra effort we may allow $A(t), \mathbf{f}(t)$ and $\mathbf{y}(t)$ (and/or their derivatives) to have a finite number of jump discontinuities when two-point schemes are employed. The details of this device are contained in Keller [5] so we do not elaborate on it

* Received by the editors July 24, 1974.

$\dagger$ Applied Mathematics, California Institute of Technology 101-50, Pasadena, California 91109. This work was supported by the U.S. Atomic Energy Commission under Contract AT(04-3-767), Project Agreement 12.

¥ Computing Science Department, University of Texas, Austin, Texas 78712 . 
here. Multipoint boundary conditions of the form

$$
\mathscr{B} \mathbf{y} \equiv \sum_{v=1}^{m} B_{v} \mathbf{y}\left(\tau_{v}\right)=\boldsymbol{\beta}, \quad a \leqq \tau_{1}<\tau_{2}<\cdots<\tau_{m} \leqq b,
$$

are also easily included.

The nonlinear problems are of the form

$$
\begin{gathered}
\mathscr{N} \mathbf{y} \equiv \mathbf{y}^{\prime}-\mathbf{f}(t, \mathbf{y})=\mathbf{0}, \quad a \leqq t \leqq b, \\
\mathbf{g}(\mathbf{y}(a), \mathbf{y}(b))=\mathbf{0},
\end{gathered}
$$

where the $n$-vectors $\mathbf{f}(t, \mathbf{y})$ and $\mathbf{g}(\mathbf{v}, \mathbf{w})$ are assumed to have sufficient smoothness. We will be concerned only with isolated solutions of (1.2), that is, solutions $\mathbf{y}=\mathbf{y}(t)$ for which the linearized problem

$$
\begin{gathered}
\mathscr{L}[\mathbf{y}] \mathbf{z}=\mathbf{0}, \quad a \leqq t \leqq b, \\
\mathscr{B}[\mathbf{y}] \mathbf{z}=\mathbf{0}
\end{gathered}
$$

has only the trivial solution $\mathbf{z}(t) \equiv \mathbf{0}$. Here $\mathscr{L}[\mathbf{y}]$ and $\mathscr{B}[\mathbf{y}]$ are as defined in (1.1) but with the matrices

$$
A(t) \equiv A(t, \mathbf{y}(t)) \equiv \frac{\partial \mathbf{f}(t, \mathbf{y}(t))}{\partial \mathbf{y}}, \quad B_{x} \equiv B_{x}[\mathbf{y}] \equiv \frac{\partial \mathbf{g}(\mathbf{y}(a), \mathbf{y}(b))}{\partial \mathbf{y}(x)}, \quad x=a, b .
$$

Again we can easily include more general boundary conditions, for example, the multipoint form

$$
\mathbf{g}\left(\mathbf{y}\left(\tau_{1}\right), \mathbf{y}\left(\tau_{2}\right), \cdots, \mathbf{y}\left(\tau_{m}\right)\right)=\mathbf{0}
$$

The difference schemes employ arbitrary families of nets, say $\left\{t_{j}\right\}$, with

$$
t_{0}=a, \quad t_{j}=t_{j-1}+h_{j}, \quad 1 \leqq j \leqq J, \quad t_{J}=b,
$$

and are subject only to the restriction that for some fixed $r>0$,

$$
h \equiv \max _{j} h_{j} \leqq r \min _{k} h_{k} .
$$

If $\mathbf{u}^{h} \equiv\left(\mathbf{u}_{0}^{T}, \mathbf{u}_{1}^{T}, \cdots, \mathbf{u}_{J}^{T}\right)^{T}$ is to approximate $\mathbf{y}^{h} \equiv\left(\mathbf{y}^{T}\left(t_{0}\right), \mathbf{y}^{T}\left(t_{1}\right), \cdots, \mathbf{y}^{T}\left(t_{J}\right)\right)^{T}$ for the linear problem (1.1), then our general difference schemes are formulated as

$$
\begin{gathered}
\mathscr{L}_{h} \mathbf{u}_{j} \equiv \sum_{k=0}^{J} C_{j k}(h) \mathbf{u}_{k}=\mathbf{F}_{j}(h ; \mathbf{f}), \quad 1 \leqq j \leqq J, \\
\mathscr{B}_{h} \mathbf{u}^{h} \equiv B_{a} \mathbf{u}_{0}+B_{b} \mathbf{u}_{J}=\boldsymbol{\beta}
\end{gathered}
$$

For the nonlinear problem (1.2), our general difference schemes are formulated as

$$
\begin{gathered}
\mathscr{N}_{h} \mathbf{u}_{j} \equiv \mathbf{G}_{j}\left(\mathbf{u}^{h}\right)=\mathbf{0}, \quad 1 \leqq j \leqq J, \\
\mathbf{g}\left(\mathbf{u}_{0}, \mathbf{u}_{J}\right)=\mathbf{0} .
\end{gathered}
$$

The linearized difference equations obtained by linearizing (1.6) about $\mathbf{u}^{h}$ are

$$
\mathscr{L}_{h}\left[\mathbf{u}^{h}\right] \mathbf{v}_{j} \equiv \sum_{k=0}^{J} C_{j k}\left(h, \mathbf{u}^{h}\right) \mathbf{v}_{k}=\mathbf{0}, \quad 1 \leqq j \leqq J,
$$




$$
\mathscr{B}_{h}\left[\mathbf{u}^{h}\right] \mathbf{v}^{h} \equiv B_{a}\left[\mathbf{u}^{h}\right] \mathbf{v}_{0}+B_{b}\left[\mathbf{u}^{h}\right] \mathbf{v}_{J}=0,
$$

where the $n \times n$ coefficient matrices are defined by

$$
\begin{aligned}
& C_{j k}\left(h, \mathbf{u}^{h}\right) \equiv \frac{\partial \mathbf{G}_{j}\left(\mathbf{u}^{h}\right)}{\partial \mathbf{u}_{k}}, \quad 0 \leqq j, k \leqq J, \\
& B_{a}\left[\mathbf{u}^{h}\right] \equiv \frac{\partial \mathbf{g}\left(\mathbf{u}_{0}, \mathbf{u}_{J}\right)}{\partial \mathbf{u}_{0}}, \quad B_{b}\left[\mathbf{u}^{h}\right] \equiv \frac{\partial \mathbf{g}\left(\mathbf{u}_{0}, \mathbf{u}_{J}\right)}{\partial \mathbf{u}_{J}}
\end{aligned}
$$

The general results of this paper are extensions of the work in [5] for linear problems and [7] for nonlinear problems. A form of these extensions is contained in the thesis of A. B. White [11]. An abstract form of the general technique used for the nonlinear case is given in [8].

2. Linear boundary value problems. For our basic theory, we need a result relating linear initial value problems and linear boundary value problems. However, it is simpler and more elegant to present the corresponding result for pairs of boundary value problems. Thus we consider first the pair of linear twopoint problems $B V(v)$ for $v=0,1$ :

$$
\begin{array}{rlrl}
\mathscr{L} \mathbf{y}^{(v)}(t) & \equiv \frac{d \mathbf{y}_{(t)}^{(v)}}{d t}-A(t) \mathbf{y}^{(v)}(t)=\mathbf{f}(t), \quad a<t<b, & \\
\mathscr{B}^{(v)} \mathbf{y}^{(v)} \equiv B_{a}^{(v)} \mathbf{y}^{(v)}(a)+B_{b}^{(v)} \mathbf{y}^{(v)}(b)=\boldsymbol{\beta}, & v=0,1 .
\end{array}
$$

These problems differ only in the matrices $B_{a}^{(v)}$ and $B_{b}^{(v)}$ that occur in the boundary conditions. Note that for all of our analysis $v$ could just as well be a continuous parameter, say in $0 \leqq v \leqq 1$, and thus our results apply to families of boundary value problems. We also define the corresponding pair of fundamental solutions, $Y^{(v)}(t)$, as the $n \times n$ matrix solutions of

$$
\begin{array}{cc}
\mathscr{L} Y^{(v)}(t)=0, & a<t<b, \\
\mathscr{B}^{(v)} Y^{(v)}=I, & v=0,1 .
\end{array}
$$

An interesting equivalence theorem relating these problems is the following theorem.

THEOREM 2.3. Let $B V(0)$ have a unique solution. Then $B V(1)$ has a unique solution if and only if $\mathscr{B}^{(1)} Y^{(0)}$ is nonsingular.

Proof. Clearly we need only show that the homogeneous boundary value problem

$$
\mathscr{L} \mathbf{y}(t)=\mathbf{0}, \quad a<t<b, \quad \mathscr{B}^{(1)} \mathbf{y}=\mathbf{0}
$$

has only the trivial solution if and only if $\mathscr{B}^{(1)} Y^{(0)}$ is nonsingular. However, every solution $\mathbf{y}(t)$ of $\mathscr{L} \mathbf{y}=\mathbf{0}$ has a unique representation of the form

$$
\mathbf{y}(t)=Y^{(0)}(t) \boldsymbol{\xi}
$$

for some $\xi \in E^{n}$. Indeed if $\mathscr{L} \mathbf{y}=\mathbf{0}$, then $\mathbf{y}(t)=\mathbf{y}^{(0)}(t)$ is the solution of $B V(0)$ with $\mathbf{f}(t) \equiv \mathbf{0}$ and $\boldsymbol{\beta} \equiv \mathscr{B}^{(0)} \mathbf{y}$. By hypothesis, this is the only such solution of $B V(0)$. But $Y^{(0)}(t) \boldsymbol{\beta}$ is also a solution of this problem, and so the unique representation 
(2.5) is established. Now $\mathbf{y}(t)$ in (2.5) is a solution of (2.4) if and only if

$$
\mathscr{B}^{(1)} \mathbf{y}=\mathscr{B}^{(1)} Y^{(0)} \boldsymbol{\xi}=\mathbf{0} .
$$

Our result now follows since $\boldsymbol{\xi}=\mathbf{0}$ is the only possibility if and only if $\mathscr{B}^{(1)} Y^{(0)}$ is nonsingular.

The result we actually need is a simple consequence of Theorem 2.3 and the uniqueness theorem for initial value problems, namely, Corollary 2.6.

Corollary 2.6. Let $B V(0)$ have a unique solution. Then $Y^{(0)}(a)$ is nonsingular.

Proof. With the choice $B_{a}^{(1)} \equiv I, B_{b}^{(1)} \equiv 0$, we see that $B V(1)$ becomes the initial value problem, the uniqueness of whose solutions is well known. Now apply Theorem 2.3 .

Finally, we point out that in all the above results we have not used the explicit form of the boundary conditions but merely the linearity of the boundary operators $\mathscr{B}^{(v)}$. Thus our results apply to any linear constraints which take $\mathbf{y}(t), a \leqq t \leqq b$, into $E^{n}$. Obviously, this includes multipoint conditions of the form

$$
\mathscr{B}^{(v)} \mathbf{y} \equiv \sum_{i=1}^{N} B_{i}^{(v)} \mathbf{y}\left(\tau_{i}\right), \quad a \leqq \tau_{1}<\tau_{2}<\cdots<\tau_{N}=b,
$$

where the $B_{i}^{(v)}$ are $n \times n$ matrices.

3. Difference methods for linear boundary value problems. The standard notions of truncation errors, consistency and stability for the scheme (1.5) applied to (1.1) can be defined as follows .

DEFINITION 3.1. (a) The truncation errors in scheme (1.5) applied to (1.1) are

$$
\begin{aligned}
\tau_{j}\{\mathbf{y}\} & \equiv \mathscr{L}_{h} \mathbf{y}\left(t_{j}\right)-\mathbf{F}_{j}(h, \mathbf{f}), \quad 1 \leqq j \leqq J, \\
\tau_{0}\{\mathbf{y}\} & \equiv \mathscr{B}_{h} \mathbf{y}-\boldsymbol{\beta},
\end{aligned}
$$

where $\mathbf{y}(t)$ is any solution of (1.1).

(b) The scheme (1.5) is consistent (accurate) of order $p$ with (for) (1.1) provided there exist constants $K_{0}>0$ and $h_{0}>0$ such that

$$
\left\|\tau_{j}\{\mathbf{y}\}\right\| \leqq K_{0} h^{p}, \quad 0 \leqq j \leqq J
$$

for all nets (1.4) with $h \leqq h_{0}$ and for all solutions $\mathbf{y}(t)$ of (1.1).

(c) The scheme (1.5) is stable provided there exist positive constants $K_{1}, K_{2}$ and $h_{0}$ such that for any net function $\mathbf{v}^{h}$ defined on (1.4) and for all $h \leqq h_{0}$

$$
\left\|\mathbf{v}_{j}\right\| \leqq K_{1}\left\|\mathscr{B}_{h} \mathbf{v}^{h}\right\|+K_{2} \max _{1 \leqq i \leqq J}\left\|\mathscr{L}_{h} \mathbf{v}_{i}\right\|, \quad 0 \leqq j \leqq J .
$$

From these definitions we easily obtain the following well-known convergence theorem.

THEOREM 3.2. If (1.5) is stable and consistent of order $p$ for (1.1), then for all nets (1.4) with $h \leqq h_{0}$,

$$
\left\|\mathbf{y}\left(t_{j}\right)-\mathbf{u}_{j}\right\| \leqq K_{0} K_{2} h^{p}
$$

that is, the scheme (1.5) is convergent of order $p$ for (1.1). Here $\mathbf{y}(t)$ is a solution of (1.1) and $\mathbf{u}^{h}$ is the solution of (1.5). 
Proof. Let $\mathbf{v}_{j} \equiv \mathbf{y}\left(t_{j}\right)-\mathbf{u}_{j}$ and use the linearity of $\mathscr{L}_{h}$ to get that

$$
\mathscr{L}_{h} \mathbf{v}_{j}=\mathscr{L}_{h} \mathbf{y}\left(t_{j}\right)-\mathscr{L}_{h} \mathbf{u}_{j}=\mathscr{L}_{h} \mathbf{y}\left(t_{j}\right)-\mathbf{F}_{j}(h, \mathbf{f})=\tau_{j}\{\mathbf{y}\}, \quad 1 \leqq j \leqq J .
$$

Similarly $\mathscr{B}_{h} \mathbf{v}=0$, and the result now follows from stability.

We introduce the matrix $\mathbb{A}_{h}$ and vectors $\mathbf{U}, \mathbf{F}$ all of order $n J+n$ as

$$
\mathbb{A}_{h} \equiv\left(\begin{array}{cccc}
B_{a} & 0 & \cdots & B_{b} \\
C_{10} & C_{11} & \cdots & C_{1 J} \\
\vdots & \vdots & & \vdots \\
C_{J 0} & C_{J 1} & \cdots & C_{J J}
\end{array}\right), \quad \mathbf{U} \equiv\left(\begin{array}{c}
\mathbf{u}_{0} \\
\mathbf{u}_{1} \\
\vdots \\
\mathbf{u}_{J}
\end{array}\right), \quad \mathbf{F} \equiv\left(\begin{array}{c}
\boldsymbol{\beta} \\
\mathbf{F}_{1}(h, \mathbf{f}) \\
\vdots \\
\mathbf{F}_{J}(h, \mathbf{f})
\end{array}\right)
$$

Then the scheme (1.5) is simply

$$
\mathbb{A}_{h} \mathbf{U}=\mathbf{F}
$$

Now we have an equivalent definition of stability as in the next lemma.

LEMMA 3.4. The scheme (1.5) is stable if and only if there exist positive constants $K$ and $h_{0}$ such that for all nets (1.4) with $h \leqq h_{0}$ the family of matrices $\mathbb{A}_{h}$ are nonsingular with uniformly bounded inverses, i.e.,

$$
\left\|\mathbb{A}_{h}^{-1}\right\| \leqq K .
$$

Proof. If $\|\cdot\|_{n}$ is the norm on $E^{n}$ used in (3.1c), then we use as the norm on $E^{n J+n}:\|\mathbf{X}\|_{n J+n}=\max { }_{0 \leqq j \leqq J}\left\|\mathbf{x}_{j}\right\|_{n}$ where $\mathbf{X}=\left(\mathbf{x}_{0}^{T}, \cdots, \mathbf{x}_{J}^{T}\right)^{T}$. Using this vector norm, the induced norm on any matrix $B \equiv\left(B_{i j}\right)$ of order $n J+n$ with the $B_{i j}$ of order $n$ is given by: $\|B\|_{n J+h}=\max _{0 \leqq i \leqq J} \sum_{j=0}^{J}\left\|B_{i j}\right\|_{n}$. Here of course $\left\|B_{i j}\right\|_{n}$ is the norm induced by $\|\cdot\|_{n}$. We now drop all subscripts on norms as their arguments suffice to identify the appropriate space.

To demonstrate Lemma (3.4) suppose (3.5) holds. Then for any $\mathbf{V} \equiv\left(\mathbf{v}_{0}^{T}, \cdots\right.$, $\left.\mathbf{v}_{J}^{T}\right)^{T}$ we have

$$
\begin{aligned}
\left\|\mathbf{v}_{j}\right\| \leqq\|\mathbf{V}\| & =\left\|\mathbb{A}_{h}^{-1} \mathbb{A}_{h} \mathbf{V}\right\| \leqq K\left\|\mathbb{A}_{h} \mathbf{V}\right\| \\
& \leqq K \max \left\{\left\|\mathscr{B}_{h} \mathbf{v}\right\|, \max _{1 \leqq i \leqq J}\left\|\mathscr{L}_{h} \mathbf{v}_{i}\right\|\right\} .
\end{aligned}
$$

Thus (3.1c) follows with, say, $K_{0}=K_{1}=K$.

Now assume (3.1c) holds. It immediately follows that $\mathbb{A}_{h}$ is nonsingular since the homogeneous system $\mathbb{A}_{h} \mathbf{U}=0$ has only the trivial solution. Then each vector $\mathbf{W} \in E^{n J+n}$ can be represented in the form $\mathbf{W}=\mathbb{A}_{h} \mathbf{V}$ for some unique $\mathbf{V} \in E^{n J+n}$. However, since (3.1c) implies, for all vectors $\mathbf{V}$, that

$$
\|\mathbf{V}\| \leqq 2 \max \left(K_{1}, K_{2}\right)\left\|\mathbb{A}_{h} \mathbf{V}\right\|
$$

it immediately follows that, for all $\mathbf{W} \neq \mathbf{0}$,

$$
\left\|\mathbb{A}_{h}^{-1} \mathbf{W}\right\| /\|\mathbf{W}\| \leqq 2 \max \left(K_{1}, K_{2}\right) .
$$

Thus (3.5) holds with some $K \leqq 2 \max \left(K_{1}, K_{2}\right)$.

We present the basic stability result for difference schemes applied to the general pair of boundary value problems $B V(v)$ in (2.1). That is, we consider the two difference problems $B V_{h}(v)$ : 


$$
\begin{aligned}
\mathscr{L}_{h} u_{j}^{(v)} \equiv \sum_{k=0}^{J} C_{j k}(h) \mathbf{u}_{k}^{(v)}=\mathbf{F}_{j}(h, \mathbf{f}), \quad 1 \leqq j \leqq J, & \\
\mathscr{B}_{h}^{(v)} \mathbf{u}^{(v)} & \equiv B_{a}^{(v)} \mathbf{u}_{0}^{(v)}+B_{b}^{(v)} u_{J}^{(v)}=\boldsymbol{\beta},
\end{aligned}
$$

Note that they differ only in the boundary conditions.

THEOREM 3.8. Let each boundary value problem $B V(v), v=0,1$, have a unique solution. Then the difference scheme $B V_{h}(0)$ is stable and consistent for $B V(0)$ if and only if $B V_{h}(1)$ is stable and consistent for $B V(1)$.

Proof. The equivalence of the consistency for the two schemes is trivial since the schemes are identical when applied to any $\mathbf{u}_{j}$ with $1 \leqq j \leqq J$ and the boundary conditions in each case are exact.

To demonstrate the equivalence of stability we introduce

$$
\mathbb{A}_{h}^{(v)} \equiv\left(\begin{array}{ccccc}
B_{a}^{(v)} & 0 & \cdots & 0 & B_{b}^{(v)} \\
C_{10} & C_{11} & \cdots & & C_{1 J} \\
\vdots & \vdots & & \vdots \\
C_{J 0} & C_{J 1} & \cdots & C_{J J}
\end{array}\right), \quad v=0,1
$$

Suppose $B V_{h}(0)$ is stable. Then $\mathbb{A}_{h}^{(0)}$ is nonsingular for all $h \leqq h_{0}$ and for some $K>0$,

$$
\left\|\left(\mathbb{A}_{h}^{(0)}\right)^{-1}\right\| \leqq K
$$

We introduce $\mathbb{D}_{h}$ as

$$
\mathbb{D}_{h} \equiv \mathbb{A}_{h}^{(1)}-\mathbb{A}_{h}^{(0)}=\left(\begin{array}{ccc}
\left(B_{a}^{(1)}-B_{a}^{(0)}\right) & 0 \cdots & \left(B_{b}^{(1)}-B_{b}^{(0)}\right) \\
0 & 0 \cdots & 0 \\
\vdots & & \\
0 & 0 \cdots & 0
\end{array}\right)
$$

Then by the assumed stability of $B V_{h}^{(0)}$, we can write

$$
\mathbb{A}_{h}^{(1)}=\left[0+\mathbb{D}_{h}\left(\mathbb{A}_{h}^{(0)}\right)^{-1}\right] \mathbb{A}_{h}^{(0)}
$$

Now denote the block structure of $\left(\mathbb{A}_{h}^{(0)}\right)^{-1}$ by means of

$$
\left(\mathbb{A}_{h}^{(0)}\right)^{-1}=\left(Z_{i j}^{(0)}\right)
$$

where the $Z_{i j}^{(0)}$ are $n \times n$ matrices and $0 \leqq i, j \leqq J$. From the $j$ th "column" of blocks we obtain, since $\mathbb{A}_{h}^{(0)} \mathbb{A}_{h}^{(0)-1}=\mathbb{l}$,

$$
\mathbb{A}_{h}^{(0)}\left(\begin{array}{c}
Z_{0 j}^{(0)} \\
\vdots \\
Z_{J j}^{(0)}
\end{array}\right)=\mathbb{Q}_{j} \equiv\left(\begin{array}{c}
0 \\
\vdots \\
0 \\
I \\
0 \\
\vdots \\
0
\end{array}\right) \leftarrow j \text { th block, } \quad 0 \leqq j \leqq J .
$$


Using (3.11) and (3.10), we find that

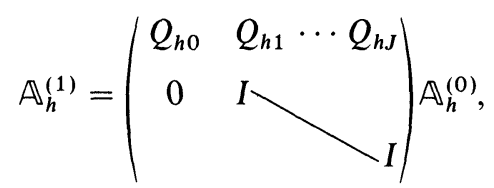

where

$$
Q_{h j} \equiv B_{a}^{(1)} Z_{0 j}^{(0)}+B_{b}^{(1)} Z_{J j}^{(0)}, \quad 0 \leqq j \leqq J .
$$

It follows from (3.12a) that $\mathbb{A}_{h}^{(1)}$ is nonsingular if and only if $Q_{h 0}$ is nonsingular. However, a glance at (3.7), (3.9) and (3.11) reveals that the $n \times n$ matrix $\left(Z_{i 0}^{(0)}\right)$ is just the difference approximation, using scheme $B V_{h}(0)$, to $Y^{(0)}\left(t_{i}\right)$, the fundamental solution for $B V(0)$ defined in (2.2). Since $B V_{h}(0)$ is stable and consistent (say, of order $p$ ), it follows from Theorem 3.2 that

$$
\left\|Y^{(0)}\left(t_{j}\right)-Z_{j 0}^{(0)}\right\|=O\left(h^{p}\right) .
$$

Then clearly

$$
\left\|\mathscr{B}^{(1)} Y^{(0)}-Q_{h 0}\right\|=O\left(h^{p}\right) .
$$

By Theorem 2.3 we have that $\mathscr{B}^{(1)} Y^{(0)}$ is nonsingular and hence the Banach lemma now implies, for $h_{0}$ sufficiently small, that $Q_{h 0}$ is nonsingular and, in fact, $\left\|Q_{h 0}^{-1}\right\| \leqq C$ for all $h \leqq h_{0}$ and some constant $C$ independent of $h$.

Thus $A_{h}^{(1)}$ is nonsingular and its inverse is

$$
\left(\mathbb{A}_{h}^{(1)}\right)^{-1}=\mathbb{A}_{h}^{(0)-1}\left(\begin{array}{ccc}
Q_{h 0}^{-1} & -Q_{h 0}^{-1} Q_{h 1} \cdots-Q_{h 0}^{-1} Q_{h J} \\
I & I
\end{array}\right)
$$

Using $\left\|Q_{h j}\right\| \leqq\left\|B_{a}^{(1)}\right\| \cdot\left\|Z_{0 j}^{(0)}\right\|+\left\|B_{b}^{(1)}\right\| \cdot\left\|Z_{J j}^{(0)}\right\|$, we obtain

$$
\sum_{j=1}^{J}\left\|Q_{h j}\right\| \leqq\left(\left\|B_{a}^{(1)}\right\|+\left\|B_{b}^{(1)}\right\|\right) K
$$

and so

$$
\left\|\left(\mathbb{A}_{h}^{(1)}\right)^{-1}\right\| \leqq K \max \left\{\|I\|, C\left[\|I\|+K\left(\left\|B_{a}^{(1)}\right\|+\left\|B_{b}^{(1)}\right\|\right)\right]\right\} .
$$

Thus the stability of $B V_{h}(1)$ follows from that of $B V_{h}(0)$.

The converse is proven by merely interchanging the superscripts $v=0$ and $v=1$ in the above arguments.

Now the relevant application of Theorem 3.8 to the scheme (1.5) applied to (1.1) is simply Corollary 3.13.

COROLlaRY 3.13. Let (1.1) have a unique solution. Then the difference scheme (1.5) is stable and consistent for (1.1) if and only if the scheme

$$
\begin{gathered}
\mathscr{L}_{h} \mathbf{v}_{j}=\mathbf{F}_{j}(h, \mathbf{f}), \quad 1 \leqq j \leqq J, \\
\mathbf{v}_{0}=\alpha
\end{gathered}
$$

is stable and consistent for the initial value problem 


$$
\begin{gathered}
\mathscr{L} \mathbf{y}=\mathbf{f}(t), \quad a<t<b, \\
\mathbf{y}(a)=\boldsymbol{\alpha} .
\end{gathered}
$$

Proof. We need simply identify (1.1) with $B V(1)$ and (3.15) with $B V(0)$. The latter clearly has a unique solution as it is just an initial value problem. Then $B V_{h}(1)$ is taken as (1.5) and $B V_{h}(0)$ is taken as (3.14). Our result follows by applying Theorem 3.8.

The schemes allowed in (1.5) are extremely general. In fact our theory now enables us to use the very well developed initial value theory of Dahlquist [1] and Henrici [2] to determine stable difference methods for linear boundary value problems. For one-step schemes, the results are particularly simple. Thus if (1.1) has a unique solution and in (1.5), we take for $j=1,2, \cdots, J$

$$
C_{j k}(h) \equiv 0 \quad \text { for } k \neq j-1, j,
$$

$$
C_{j, j-1}(h) \equiv-\frac{1}{h_{j}} I+\tilde{C}_{j, j-1}(h), \quad C_{j, j}(h) \equiv \frac{1}{h_{j}} I+\tilde{C}_{j, j}(h),
$$

then (1.5) is stable and convergent for (1.1) if (1.5) is consistent with (1.1). This result easily follows from Corollary 3.13 and Theorem 1 of Isaacson and Keller [4, p. 396], which implies the stability of (1.5) with coefficients satisfying (3.16). We point out that one-step schemes for first order systems are "compact as possible" in the terminology of Kreiss [9], and thus we obtain his results for such systems and extend them to nonuniform nets.

There are of course many schemes for initial value problems that are not treated in the above cited works. For example, the midpoint rule for initial value problems is stable, given appropriate starting data; but by altering the scheme at only one point (while not affecting consistency), it can be made unstable. Conversely some schemes which are unstable for initial value problems become stable when some of the initial data are replaced by conditions at the end of the interval. These examples, pointed out by H.-O. Kreiss, serve to stress the form in which the initial and boundary conditions are required to enter in the present theory.

Finally we note that asymptotic error expansions are easily obtained when the corresponding truncation error expansions are known by simply using the stability result. This is done in some detail for special one-step schemes in [5]. For more general schemes devised from initial value methods, we can readily employ the expansions given by Gragg [13] for one-step methods and by Henrici [2] and Engquist [12] for multistep methods.

4. Difference methods for nonlinear boundary value problems. The definitions of truncation errors, consistency and stability for the scheme (1.6) applied to (1.2) are as follows

DEFINITION 4.1. (a) The truncation errors in scheme (1.6) applied to (1.2) are

$$
\tau_{0}\{\mathbf{y}\} \equiv \mathbf{g}(\mathbf{y}(a), \mathbf{y}(b)), \quad \tau_{j}\{\mathbf{y}\} \equiv \mathscr{N}_{h} \mathbf{y}\left(t_{j}\right), \quad 1 \leqq j \leqq J,
$$

where $\mathbf{y}(t)$ is any solution of (1.2). 
(b) The scheme (1.6) is consistent (accurate) of order $p$ for the solution $\mathbf{y}(t)$ of (1.2) provided there exist constants $K_{0}>0$ and $h_{0}>0$ such that

$$
\left\|\tau_{j}\{\mathbf{y}\}\right\| \leqq K_{0} h^{p}, \quad 0 \leqq j \leqq J
$$

for all nets (1.4) with $h \leqq h_{0}$.

(c) The scheme (1.6) is stable for $\mathbf{y}^{h}$ provided there exist positive constants $K_{\rho}, \rho$ and $h_{0}$ such that for all net functions $\mathbf{v}^{h}, \mathbf{w}^{h} \in S_{\rho}\left(\mathbf{y}^{h}\right) \equiv\left\{\mathbf{u}^{h}:\left\|\mathbf{u}_{j}-\mathbf{y}_{j}\right\| \leqq \rho\right.$, $0 \leqq j \leqq J\}$ and all nets (1.4) with $h \leqq h_{0}$,

$$
\left\|\mathbf{v}_{j}-\mathbf{w}_{j}\right\| \leqq K_{\rho} \max \left\{\left\|\mathscr{N}_{h} \mathbf{v}_{k}-\mathscr{N}_{h} \mathbf{w}_{k}\right\|, 1 \leqq k \leqq J ;\left\|\mathbf{g}\left(\mathbf{v}_{0}, \mathbf{v}_{J}\right)-\mathbf{g}\left(\mathbf{w}_{0}, \mathbf{w}_{J}\right)\right\|\right\} .
$$

In analogy with Theorem 3.2, we now have the well-known Theorem 4.2.

THEOREM 4.2. Let $\mathbf{y}(t)$ be a solution of (1.2) and for all nets (1.4) with $h \leqq h_{0}$ let $\mathbf{u}^{h}$ be a solution of (1.6) in $S_{\rho}\left(\mathbf{y}^{h}\right)$ where $\mathbf{y}_{j} \equiv \mathbf{y}\left(t_{j}\right)$. If (1.6) is accurate of order $p$ for $\mathbf{y}(t)$ and stable for $\mathbf{y}^{h}$, then on all nets (1.4) with $h \leqq h_{0}$

$$
\left\|\mathbf{y}\left(t_{j}\right)-\mathbf{u}_{j}\right\| \leqq K_{0} K_{\rho} h^{p}
$$

Proof. By Definition 4.1(c) with $\mathbf{v}_{j} \equiv \mathbf{y}\left(t_{j}\right)$ and $\mathbf{w}_{j} \equiv \mathbf{u}_{j}$,

$$
\begin{aligned}
& \left\|\mathbf{y}\left(t_{j}\right)-\mathbf{u}_{j}\right\| \\
& \quad \leqq K_{\rho} \max \left\{\left\|\mathscr{N}_{h} \mathbf{y}\left(t_{k}\right)-\mathscr{N}_{h} \mathbf{u}_{k}\right\|, 1 \leqq k \leqq J ;\left\|\mathbf{g}(\mathbf{y}(a), \mathbf{y}(b))-\mathbf{g}\left(\mathbf{u}_{0}, \mathbf{u}_{J}\right)\right\|\right\} .
\end{aligned}
$$

Using $(1.2 b)$ and $(1.6 a, b)$, we get the result upon recalling Definition $4.1(a, b)$.

The basic problems are of course to insure that (1.6) has a solution in $S_{\rho}\left(\mathbf{y}^{h}\right)$ for all $h \leqq h_{0}$ and to verify stability. We could apply the general theory developed in [8] to get these results, but in the interest of completeness we indicate the details. For-stability we have the following lemma.

LEMMA 4.3. Let $\mathbf{y}(t)$ be an isolated solution of (1.2) and assume

(i) the linear difference scheme

$$
\mathscr{L}_{h}\left[\mathbf{y}^{h}\right] \mathbf{v}_{j}=0, \quad 1 \leqq j \leqq J, \quad \mathbf{v}_{0}=\mathbf{z}_{0},
$$

defined in (1.7) is stable and consistent for the initial value problem

$$
\mathscr{L}[\mathbf{y}] \mathbf{z}=0, \quad a \leqq t \leqq b, \quad \mathbf{z}(a)=\mathbf{z}_{0}:
$$

(ii) for some $\rho>0, K_{L}>0, h_{0}>0$, all $\mathbf{w}^{h} \in S_{\rho}\left(\mathbf{y}^{h}\right)$ and for all $h \leqq h_{0}$,

$$
\left\|\mathscr{L}_{h}\left[\mathbf{y}^{h}\right]-\mathscr{L}_{h}\left[\mathbf{w}^{h}\right]\right\| \leqq K_{L}\left\|\mathbf{y}^{h}-\mathbf{w}^{h}\right\|
$$

$$
\left\|B_{x}\left[\mathbf{y}^{h}\right]-B_{x}\left[\mathbf{w}^{h}\right]\right\| \leqq K_{L} \max \left\{\left\|\mathbf{y}(a)-\mathbf{w}_{0}\right\|,\left\|\mathbf{y}(b)-\mathbf{w}_{J}\right\|\right\}, \quad x=a, b
$$

Then the scheme (1.6) is stable for $\mathbf{y}^{h}$ provided $\rho$ is sufficiently small.

Proof. Define $\mathbb{A}_{h}\left[\mathbf{w}^{h}\right]$ for any $\mathbf{w}^{h} \in S_{\rho}\left(\mathbf{y}^{h}\right)$ by using $(1.7 \mathrm{c})$ with $\mathbf{u}^{h}$ replaced by $\mathbf{w}^{h}$ in (3.3). We claim that $\mathbb{A}_{h}\left[\mathbf{y}^{h}\right]$ is nonsingular and for some constant $K>0$,

$$
\left\|\mathbb{A}_{h}^{-1}\left[\mathbf{y}^{h}\right]\right\| \leqq K \text { for all } h \leqq 0,
$$

This follows from Corollary 3.13, Lemma 3.4 and $4.3(\mathrm{a}, \mathrm{b})$, since $\mathbf{y}(t)$ is assumed an isolated solution, and thus (1.3) has a unique solution. 
Let us write the nonlinear difference operators of (1.6) in the vector form

$$
\boldsymbol{\Phi}\left(\mathbf{u}^{h}\right) \equiv\left(\begin{array}{c}
\mathbf{g}\left(\mathbf{u}_{0}, \mathbf{u}_{J}\right) \\
\mathscr{N}_{h} \mathbf{u}_{1} \\
\vdots \\
\mathscr{N}_{h} \mathbf{u}_{J}
\end{array}\right) .
$$

Then by the assumed differentiability of the $\mathbf{G}_{\mathbf{j}}(\cdot)$ and $\mathbf{g}(\cdot, \cdot)$,

$$
\boldsymbol{\Phi}\left(\mathbf{v}^{h}\right)-\boldsymbol{\Phi}\left(\mathbf{w}^{h}\right)=\hat{\mathbb{A}}_{h}\left[\mathbf{v}^{h}, \mathbf{w}^{h}\right]\left(\mathbf{v}^{h}-\mathbf{w}^{h}\right),
$$

where

$$
\widehat{\mathbb{A}}_{h}\left[\mathbf{v}^{h}, \mathbf{w}^{h}\right] \equiv \int_{0}^{1} \mathbb{A}_{h}\left[s \mathbf{v}^{h}+(1-s) \mathbf{w}^{h}\right] d s .
$$

It follows from (4.4) that for all $\mathbf{v}^{h}, \mathbf{w}^{h} \in S_{\rho}\left(\mathbf{y}^{h}\right)$

$$
\left\|\hat{\mathbb{A}}\left[\mathbf{v}^{h}, \mathbf{w}^{h}\right]-\mathbb{A}_{h}\left[\mathbf{y}^{h}\right]\right\| \leqq \rho K_{L} .
$$

Thus if $\rho$ is so small that $\rho K_{L} K<1$, the Banach lemma implies $\widehat{\mathbb{A}}_{h}[\cdot, \cdot]$ nonsingular and in fact

$$
\left\|\hat{\mathbb{A}}_{h}^{-1}\left[\mathbf{v}^{h}, \mathbf{w}^{h}\right]\right\| \leqq \frac{K}{1-\rho K_{L} K}
$$

Stability as in Definition 4.1(c) is simply, using (4.6),

$$
\left\|\mathbf{v}^{h}-\mathbf{w}^{h}\right\| \leqq K_{\rho}\left\|\Phi\left(\mathbf{v}^{h}\right)-\boldsymbol{\Phi}\left(\mathbf{w}^{h}\right)\right\|
$$

and it clearly follows with $K_{\rho}=K /\left(1-\rho K_{L} K\right)$.

The existence of a unique solution in $S_{\rho}\left(\mathbf{y}^{h}\right)$ of the difference equations (1.6) for each $h \leqq h_{0}$ is established by contraction mappings applied to

$$
\mathbf{u}^{h}=\mathbf{u}^{h}-\mathbb{A}_{h}^{-1}\left[\mathbf{y}^{h}\right] \boldsymbol{\Phi}\left(\mathbf{u}^{h}\right) .
$$

The proof assumes consistency as in Definition 4.1(a,b) and the hypothesis of Lemma 4.3. The details are contained in [8, Thm. 3.6] and are similar to part of the argument in $[7, \S 3]$. Combining these results with those of Theorem 4.2 and Lemma 4.3, we have the following basic theorem.

THEOREM 4.8. Let $\mathbf{y}(t)$ be an isolated solution of (1.2). Let the difference scheme (1.6) be accurate of order $p$ for $\mathbf{y}(t)$ and satisfy the hypothesis (i) and (ii) of Lemma 4.3. Then for $\rho>0$ and $h_{0}>0$, both sufficiently small, the difference equations (1.6) have for each $h \leqq h_{0}$ a unique solution $\mathbf{u}^{h} \in S_{\rho}\left(\mathbf{y}^{h}\right)$ with

$$
\left\|\mathbf{y}\left(t_{j}\right)-\mathbf{u}_{j}\right\| \leqq M h^{p}
$$

for some constant $M>0$.

To actually compute the numerical solution, we employ Newton's method in the form

$$
\begin{gathered}
\mathbf{u}_{0}^{h} \in S_{\rho_{0}}\left(\mathbf{y}^{h}\right), \\
\mathbb{A}_{h}\left[\mathbf{u}_{v}^{h}\right]\left(\mathbf{u}_{v+1}^{h}-\mathbf{u}_{v}^{h}\right)=-\boldsymbol{\Phi}\left(\mathbf{u}_{v}^{h}\right), \quad v=0,1,2, \cdots .
\end{gathered}
$$


The quadratic convergence is easily established under the hypothesis of Theorem 4.8 with some $\rho_{0} \leqq \rho$. For any $\mathbf{v}^{h} \in S_{\rho_{0}}\left(\mathbf{y}^{h}\right)$, we have the identity

$$
\mathbb{A}_{h}\left[\mathbf{v}^{h}\right]=\mathbb{A}_{h}\left[\mathbf{y}^{h}\right]\left\{0-\mathbb{A}_{n}^{-1}\left[\mathbf{y}^{h}\right]\left(\mathbb{A}_{h}\left[\mathbf{y}^{h}\right]-\mathbb{A}_{h}\left[\mathbf{v}^{h}\right]\right)\right\} .
$$

Using $(4.4 \mathrm{a}, \mathrm{b}),(4.5)$ and the Banach lemma, we have that $A_{h}\left[\mathbf{v}^{h}\right]$ is nonsingular with

$$
\left\|\mathbb{A}_{h}^{-1}\left[\mathbf{v}^{h}\right]\right\| \leqq K_{\rho_{0}}=\frac{K}{1-\rho_{0} K_{L} K} .
$$

From (4.9b) with $v=0$ we obtain, using (4.7),

$$
\mathbf{u}_{1}^{h}-\mathbf{u}_{0}^{h}=-\mathbb{A}_{h}^{-1}\left[\mathbf{u}_{0}^{h}\right] \boldsymbol{\Phi}\left(\mathbf{y}^{h}\right)+\mathbb{A}_{h}^{-1}\left[\mathbf{u}_{0}^{h}\right] \widehat{\mathbb{A}}_{h}\left[\mathbf{u}_{0}^{h}, \mathbf{y}^{h}\right]\left(\mathbf{y}^{h}-\mathbf{u}_{0}^{h}\right) .
$$

Now note that

$$
\mathbb{A}_{h}^{-1}\left[\mathbf{u}_{0}^{h}\right] \widehat{\mathbb{A}}_{h}\left[\mathbf{u}_{0}^{h}, \mathbf{y}^{h}\right] \equiv \mathbb{0}+\mathbb{A}_{h}^{-1}\left[\mathbf{u}_{0}^{h}\right]\left(\widehat{\mathbb{A}}_{h}\left[\mathbf{u}_{0}^{h}, \mathbf{y}^{h}\right]-\mathbb{A}_{h}\left[\mathbf{u}_{0}^{h}\right]\right) .
$$

Using (4.10) and the Lipschitz continuity (4.4), we find that for any $\rho_{0} \leqq \rho$ there exists some $C>0$ such that

$$
\left\|\mathbb{A}_{h}^{-1}\left[\mathbf{u}_{0}^{h}\right] \hat{\mathbb{A}}_{h}\left[\mathbf{u}_{0}^{h}, \mathbf{y}^{h}\right]\right\| \leqq C .
$$

Thus we finally get, recalling $(4.1 \mathrm{a}, \mathrm{b})$, that

$$
\left\|\mathbf{u}_{1}^{h}-\mathbf{u}_{0}^{h}\right\| \leqq K_{\rho_{0}} K_{0} h^{p}+C \rho_{0} .
$$

From (4.10) and (4.11), the quadratic convergence of Newton's method follows in standard fashion (see [6] or [10]). The convergence proof in [7] is unnecessarily restrictive, as has been observed by $F$. de Hoog [3], since sharp estimates of $\left\|\boldsymbol{\Phi}\left(\mathbf{u}_{0}^{h}\right)\right\|$ were sought rather than of $\left\|\mathbb{A}_{h}^{-1}\left[\mathbf{u}_{0}^{h}\right] \boldsymbol{\Phi}\left(\mathbf{u}_{0}^{h}\right)\right\|$ as we do above. In particular, we stress that it is not necessary that $\rho_{0}$ be reduced with $h$, and thus a much larger sphere is shown to be in the domain of attraction for the root in question.

In closing, we note that the complete nonlinear theory goes over for the general nonlinear multipoint boundary conditions. The details are quite similar to those contained in the Appendix to [7] and so we do not repeat them here.

\section{REFERENCES}

[1] G. DAHLQuist, Convergence and stability in the numerical integration of ordinary differential equations, Math. Scand., 4 (1956), pp. 33-53.

[2] P. Henrici, Discrete Variable Methods in Ordinary Differential Equations, John Wiley, New York, 1962.

[3] F. DE Hoog, Private communication, 1974.

[4] E. Isaacson and H. B. Keller, Analysis of Numerical Methods, John Wiley, New York, 1966.

[5] H. B. KELLER, Accurate difference methods for linear ordinary differential systems subject to linear constraints, this Journal, 6 (1969), pp. 8-30.

[6] - Newton's method under mild differentiability conditions, J. Comput. System Sci., 4 (1970), pp. 15-28.

[7] - Accurate difference methods for nonlinear two-point boundary value problems, this Journal, 11 (1974), pp. 305-320.

[8] - Approximation methods for nonlinear problems with application to two-point boundary value problems, Math. Comp., to appear (1975).

[9] H.-O. KREISS, Difference approximations for boundary and eigenvalue problems for ordinary differential equations, Math. Comp., 26 (1972), pp. 605-624. 
[10] J. ORtega AND W. Rheinboldt, Iterative Solution of Nonlinear Equations in Several Variables, Academic Press, New York, 1970.

[11] A. B. White, Numerical solution of two-point boundary value problems, Thesis, Calif. Inst. of Tech., Pasadena, Calif., 1974.

[12] B. EngQuist, Asymptotic error expansions for multistep methods, Research Rep., Computer Science Dept., Uppsala University, Uppsala, Sweden, 1969.

[13] W. B. GRAGG, On extrapolation algorithms for ordinary initial value problems, this Journal, 2(1965), pp. $384-403$. 